\title{
A heuristic for global nonlinear optimization
}

\author{
Michel Bierlaire
}

in collaboration with Michaël Thémans (Nestlé Research Center) and Nicolas Zufferey (U. Geneva)

Transport and Mobility Laboratory, Ecole Polytechnique Fédérale de Lausanne 


\section{Global optimization}

Motivation: estimation of econometric models

- (Conditional) Maximum Likelihood estimation of MEV models

- More advanced models:

- continuous and discrete mixtures of MEV models

- estimation with panel data

- latent classes

- latent variables

- discrete-continuous models

- etc... 


\section{Global optimization}

Objective: identify the global minimum of

$$
\min _{x \in \mathbb{R}^{n}} f(x),
$$

where

- $f: \mathbb{R}^{n} \rightarrow \mathbb{R}$ is twice differentiable.

- No special structure is assumed on $f$. 


\section{Literature}

Local nonlinear optimization:

- Main focus:

- global convergence

- towards a local minimum

- with fast local convergence.

- Vast literature

- Efficient algorithms

- Softwares 


\section{Literature}

Global nonlinear optimization: exact approaches

- Real algebraic geometry (representation of polynomials, semidefinite programming)

- Interval arithmetic

- Branch \& Bound

- DC - difference of convex functions 


\section{Literature}

Global nonlinear optimization: heuristics

- Usually hybrid between derivative-free methods and heuristics from discrete optimization. Examples:

- Glover (1994) Tabu + scatter search

- Franze and Speciale (2001) Tabu + pattern search

- Hedar and Fukushima (2004) Sim. annealing + pattern

- Hedar and Fukushima (2006) Tabu + direct search

- Mladenovic et al. (2006) Variable Neighborhood search (VNS) 


\section{Our heuristic}

Framework: VNS

Ingredients:

1. Local search

$$
\left(\operatorname{SUCCESS}, y^{*}\right) \leftarrow \operatorname{LS}\left(y_{1}, \ell_{\max }, \mathcal{L}\right),
$$

where

- $y_{1}$ is the starting point

- $\ell_{\max }$ is the maximum number of iterations

- $\mathcal{L}$ is the set of already visited local optima

- Algorithm: trust region 


\section{Our heuristic}

1. Local search

$$
\left(\operatorname{SUCCESS}, y^{*}\right) \leftarrow \operatorname{LS}\left(y_{1}, \ell_{\max }, \mathcal{L}\right),
$$

- If $\mathcal{L} \neq \emptyset$, LS may be interrupted prematurely

- If $\mathcal{L}=\emptyset$, LS runs toward convergence

- If local minimum identified, SUCCESS=true 


\section{Our heuristic}

2. Neighborhood structure

- Neighborhoods: $\mathcal{N}_{k}(x), k=1, \ldots, n_{\max }$

- Nested structure: $\mathcal{N}_{k}(x) \subset \mathcal{N}_{k+1}(x) \subseteq \mathbb{R}^{n}$, for each $k$

- Neighbors generation

$$
\left(z_{1}, z_{2}, \ldots, z_{p}\right)=\operatorname{NEIGHBORS}(x, k) .
$$

- Typically, $n_{\max }=5$ and $p=5$. 


\section{The VNS framework}

Initialization $x_{1}^{*}$ local minimum of $f$

- Cold start: run LS once

- Warm start: run LS from randomly generated starting points

Stopping criteria Interrupt if

1. $k>n_{\text {max }}$ : the last neighborhood has been unsuccessfully investigated

2. CPU time $\geq t_{\max }$, typ. 30 minutes (18K seconds).

3. Number of function evaluations $\geq$ eval $_{\max }$, typ. $10^{5}$. 


\section{The VNS framework}

\section{Main loop Steps:}

1. Generate neighbors of $x_{\text {best }}^{k}$ :

$$
\left(z_{1}, z_{2}, \ldots, z_{p}\right)=\operatorname{NEIGHBORS}\left(x_{\text {best }}^{k}, k\right) .
$$

2. Apply the $p$ local search procedures:

$$
\left(\operatorname{SUCCESS}_{j}, y_{j}^{*}\right) \leftarrow \operatorname{LS}\left(z_{j}, \ell_{\text {large }}, \mathcal{L}\right) .
$$

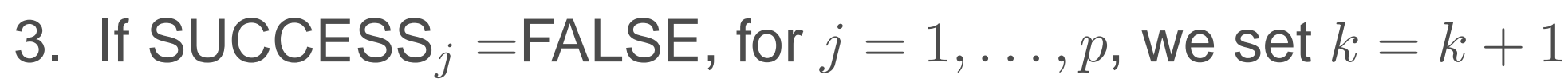
and proceed to the next iteration. 


\section{The VNS framework}

Main loop Steps (ctd):

4. Otherwise,

$$
\mathcal{L}=\mathcal{L} \cup\left\{y_{j}^{*}\right\} .
$$

for each $j$ such that SUCCESS SU $_{j}=$ TRUE

5. Define $x_{\text {best }}^{k+1}$

$$
f\left(x_{\text {best }}^{k+1}\right) \leq f(x), \text { for each } x \in \mathcal{L} .
$$

6. If $x_{\text {best }}^{k+1}=x_{\text {best }}^{k}$, no improvement. We set $k=k+1$ and proceed to the next iteration. 


\section{The VNS framework}

Main loop Steps (ctd):

7. Otherwise, we have found a new candidate for the global optimum. The neighborhood structure is reset, we set $k=1$ and proceed to the next iteration.

Output The output is the best solution found during the algorithm,

that is $x_{\text {best }}^{k}$. 


\section{Local search}

- Classical trust region method with quasi-newton update

- Key feature: premature interruption

- Three criteria: we check that

1. the algorithm does not get too close to an already identified local minimum.

2. the gradient norm is not too small when the value of the objective function is far from the best.

3. a significant reduction in the objective function is achieved. 


\section{Neighborhoods}

The key idea: analyze the curvature of $f$ at $x$

- Let $v_{1}, \ldots, v_{n}$ be the (normalized) eigenvectors of $H$

- Let $\lambda_{1}, \ldots, \lambda_{n}$ be the eigenvalues.

- Define direction $w_{1}, \ldots, w_{2 n}$, where $w_{i}=v_{i}$ if $i \leq n$, and $w_{i}=-v_{i}$ otherwise.

- Size of the neighborhood: $d_{1}=1, d_{k}=1.5 d_{k-1}, k=2, \ldots$. 


\section{Neighborhoods}

- Neighbors:

$$
z_{j}=x+\alpha d_{k} w_{i}, \quad j=1, \ldots, p,
$$

where

- $\alpha$ is randomly drawn $U[0.75,1]$

- $i$ is a selected index

- Selection of $w_{i}$ :

- Prefer directions where the curvature is larger

- Motivation: better potential to jump in the next valley 


\section{Neighborhoods: selection of $w_{i}$}

$$
P\left(w_{i}\right)=P\left(-w_{i}\right)=\frac{e^{\beta \frac{\left|\lambda_{i}\right|}{d_{k}}}}{2 \sum_{j=1}^{n} e^{\beta \frac{\left|\lambda_{j}\right|}{d_{k}}}} .
$$

- In large neighborhoods ( $d_{k}$ large), curvature is less relevant and probabilities are more balanced.

- We tried $\beta=0.05$ and $\beta=0$.

- The same $w_{i}$ can be selected more than once

- The random step $\alpha$ is designed to generate different neighbors in this case 


\section{Numerical results}

- 25 problems from the literature

- Dimension from 2 to 100

- Most with several local minima

- Some with "crowded" local minima

- Measures of performance:

1. Percentage of success (i.e. identification of the global optimum) on 100 runs

2. Average number of function evaluations for successful runs 


\section{Shubert function}

$\left(\sum_{j=1}^{5} j \cos \left((j+1) x_{1}+j\right)\right)\left(\sum_{j=1}^{5} j \cos \left((j+1) x_{2}+j\right)\right)$

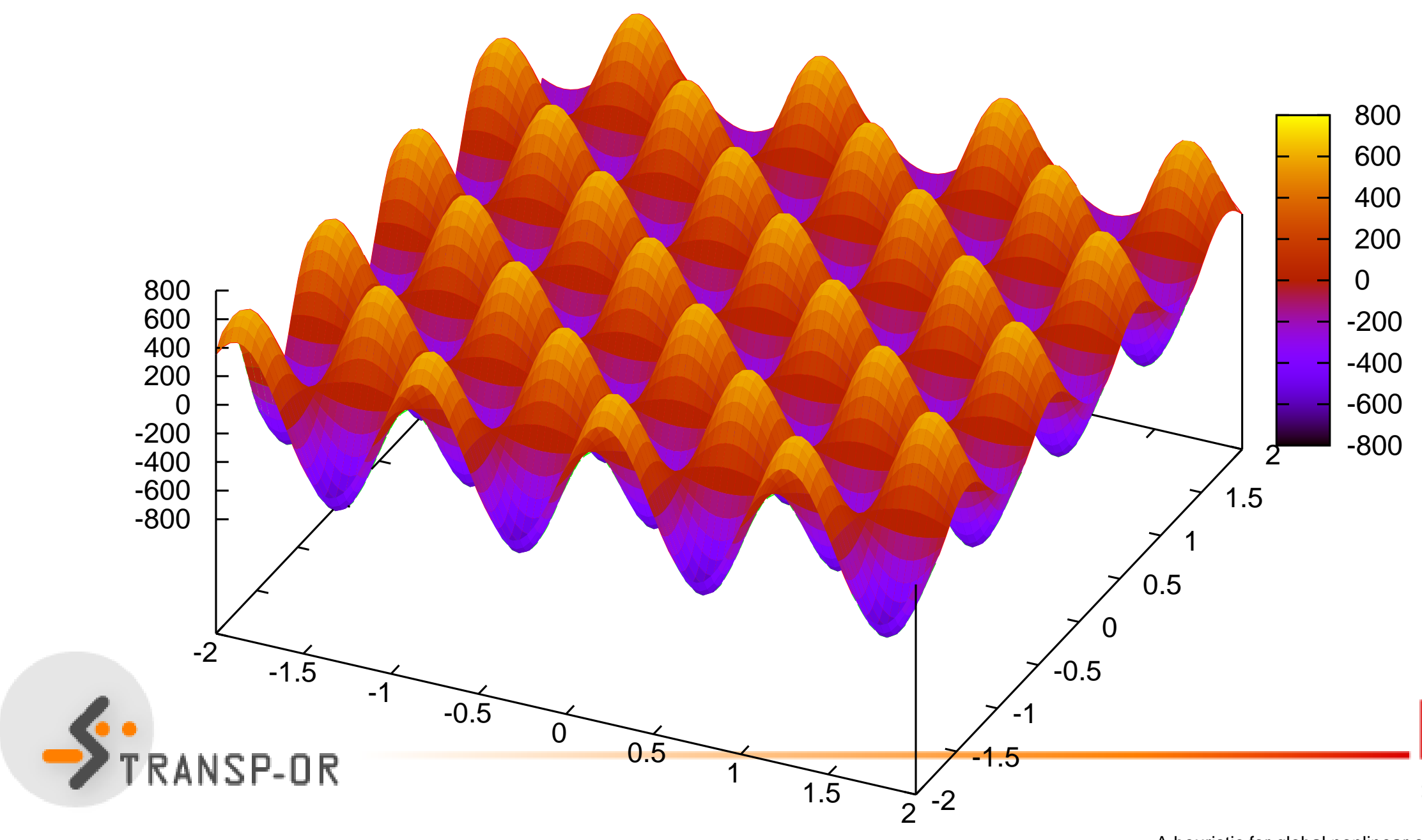




\section{Numerical results}

Competition:

1. Direct Search Simulated Annealing (DSSA) Hedar \& Fukushima (2002).

2. Continuous Hybrid Algorithm (CHA) Chelouah \& Siarry (2003).

3. Simulated Annealing Heuristic Pattern Search (SAHPS) Hedar \& Fukushima (2004).

4. Directed Tabu Search (DTS) Hedar \& Fukushima (2006) .

5. General variable neighborhood search (GVNS) Mladenovic et al. (2006) 


\section{Numerical results: success rate}

\begin{tabular}{|c|c|c|c|c|c|c|}
\hline Problem & VNS & $\mathrm{CHA}$ & DSSA & DTS & SAHPS & GVNS \\
\hline $\mathrm{RC}$ & 100 & 100 & 100 & 100 & 100 & 100 \\
\hline ES & 100 & 100 & 93 & 82 & 96 & \\
\hline $\mathrm{RT}$ & 84 & 100 & 100 & & 100 & \\
\hline $\mathrm{SH}$ & 78 & 100 & 94 & 92 & 86 & 100 \\
\hline$R_{2}$ & 100 & 100 & 100 & 100 & 100 & 100 \\
\hline$Z_{2}$ & 100 & 100 & 100 & 100 & 100 & \\
\hline DJ & 100 & 100 & 100 & 100 & 100 & \\
\hline$H_{3,4}$ & 100 & 100 & 100 & 100 & 95 & 100 \\
\hline$S_{4,5}$ & 100 & 85 & 81 & 75 & 48 & 100 \\
\hline$S_{4,7}$ & 100 & 85 & 84 & 65 & 57 & \\
\hline$S_{4}^{\circ} T^{\circ}$ & 100 & 85 & 77 & 52 & 48 & 100 \\
\hline
\end{tabular}




\section{Numerical results: success rate}

\begin{tabular}{|c|c|c|c|c|c|c|}
\hline Problem & VNS & $\mathrm{CHA}$ & DSSA & DTS & SAHPS & GVNS \\
\hline$R_{5}$ & 100 & 100 & 100 & 85 & 91 & \\
\hline$Z_{5}$ & 100 & 100 & 100 & 100 & 100 & \\
\hline$H_{6,4}$ & 100 & 100 & 92 & 83 & 72 & 100 \\
\hline$R_{10}$ & 100 & 83 & 100 & 85 & 87 & 100 \\
\hline$Z_{10}$ & 100 & 100 & 100 & 100 & 100 & \\
\hline $\mathrm{HM}$ & 100 & & 100 & & & \\
\hline$G R_{6}$ & 100 & & 90 & & & \\
\hline$G R_{10}$ & 100 & & & & & 100 \\
\hline $\mathrm{CV}$ & 100 & & 100 & & & \\
\hline DX & 100 & & 100 & & & \\
\hline GRANS & $0 k^{00}$ & & & & & 100 \\
\hline
\end{tabular}




\section{Numerical results: success rate}

\begin{tabular}{|l|c|c|c|c|c|c|}
\hline Problem & VNS & CHA & DSSA & DTS & SAHPS & GVNS \\
\hline$R_{50}$ & 100 & 79 & & 100 & & \\
$Z_{50}$ & 100 & 100 & & 0 & & \\
$R_{100}$ & 100 & 72 & & 0 & & \\
\hline
\end{tabular}

- Excellent success rate on these problems

- Best competitor: GVNS (Mladenovic et al, 2006) 


\section{Performance Profile}

$\rightarrow$ Performance Profile proposed by Dolan and Moré (2002)

Algorithms Problems

\begin{tabular}{l|llllllllll} 
Method A & 20 & 10 & $* *$ & 10 & $* *$ & 20 & 10 & 15 & 25 & $* *$ \\
Method B & 10 & 30 & 70 & 60 & 70 & 80 & 60 & 75 & $* *$ & $* *$
\end{tabular}




\section{Performance Profile}

$\rightarrow$ Performance Profile proposed by Dolan and Moré (2002)

Algorithms Problems

\begin{tabular}{l|cccccccccc} 
Method A & 2 & 1 & $r_{\text {fail }}$ & 1 & $r_{\text {fail }}$ & 1 & 1 & 1 & 1 & $r_{\text {fail }}$ \\
Method B & 1 & 3 & 1 & 6 & 1 & 4 & 6 & 5 & $r_{f a i l}$ & $r_{f a i l}$
\end{tabular}




\section{Performance Profile}

$\rightarrow$ Performance Profile proposed by Dolan and Moré (2002)

Algorithms Problems

\begin{tabular}{l|cccccccccc}
\hline Method A & 2 & 1 & $r_{\text {fail }}$ & 1 & $r_{\text {fail }}$ & 1 & 1 & 1 & 1 & $r_{\text {fail }}$ \\
Method B & 1 & 3 & 1 & 6 & 1 & 4 & 6 & 5 & $r_{\text {fail }}$ & $r_{\text {fail }}$
\end{tabular}

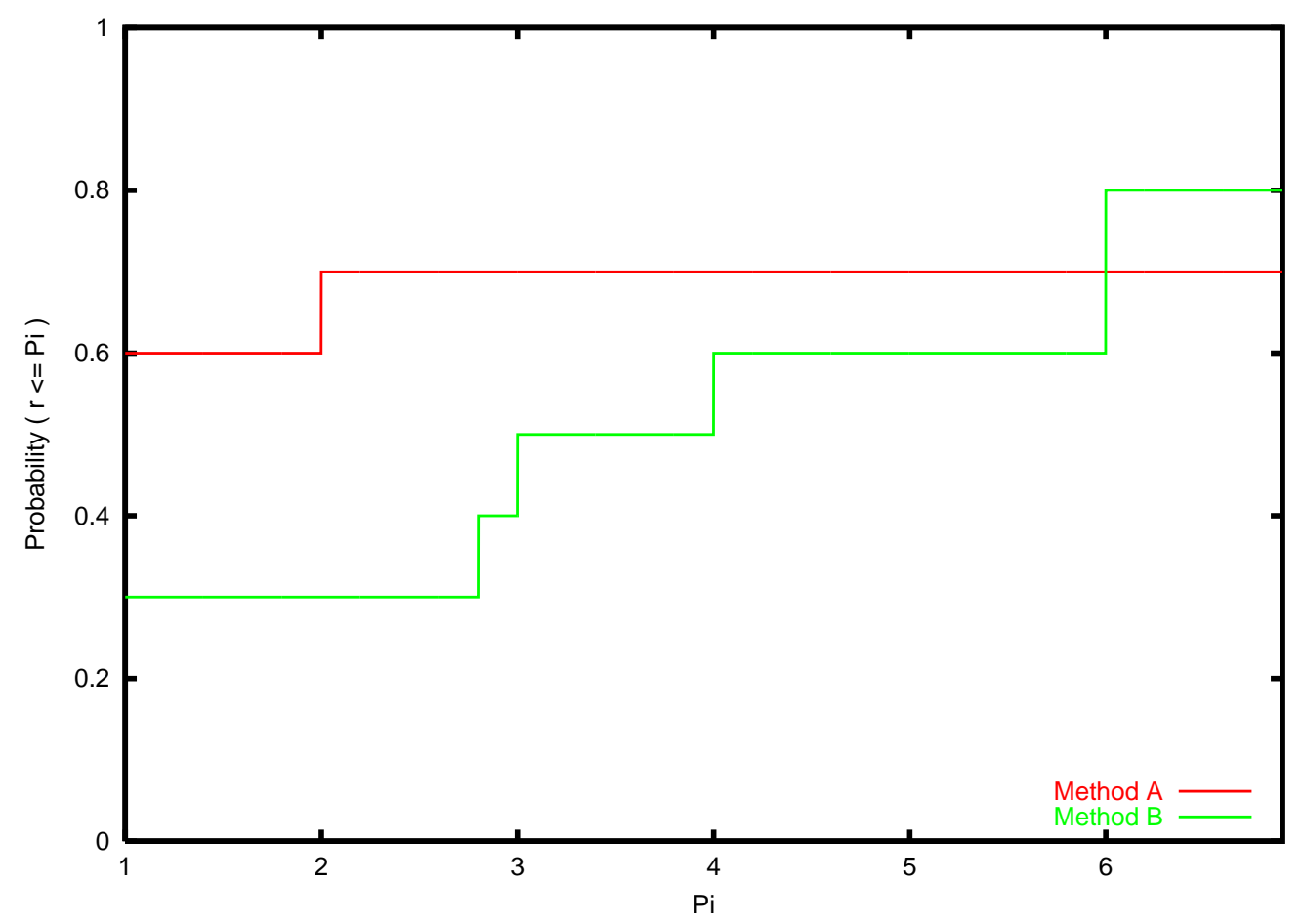




\section{Numerical results: efficiency}

Number of function evaluations (4 competitors)

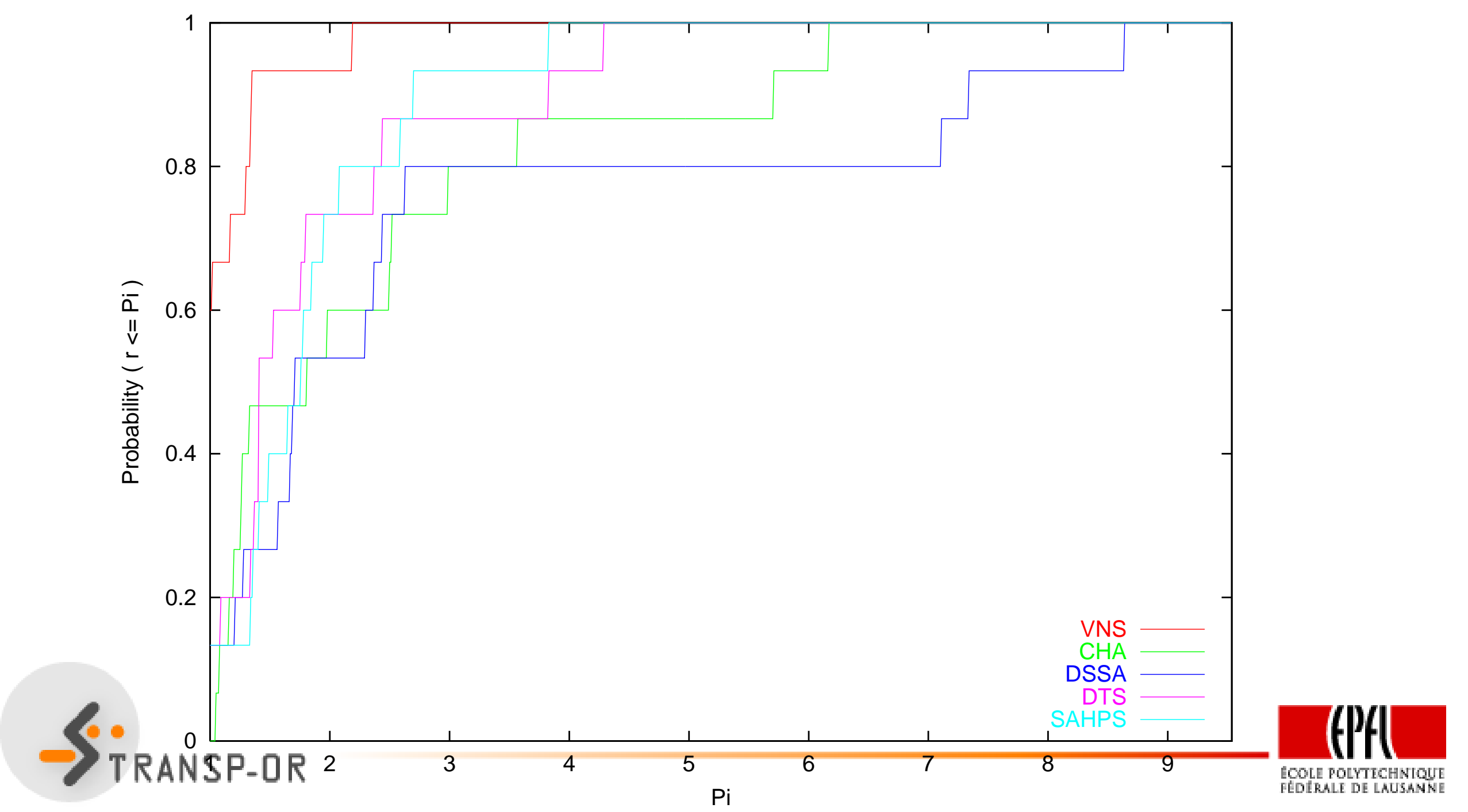




\section{Numerical results: efficiency}

Number of function evaluations (zoom)

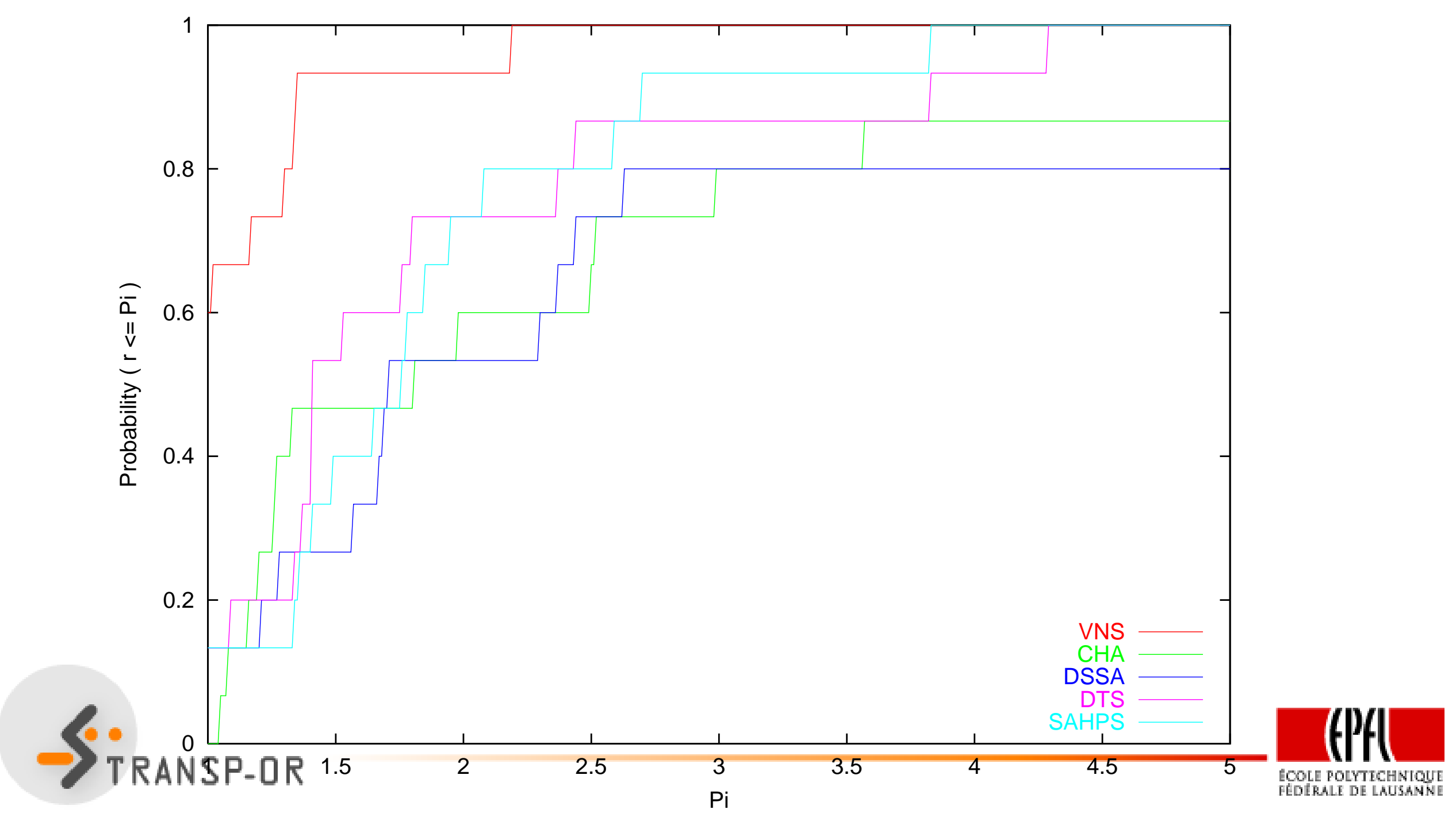




\section{Numerical results: efficiency}

Number of function evaluations (GVNS)

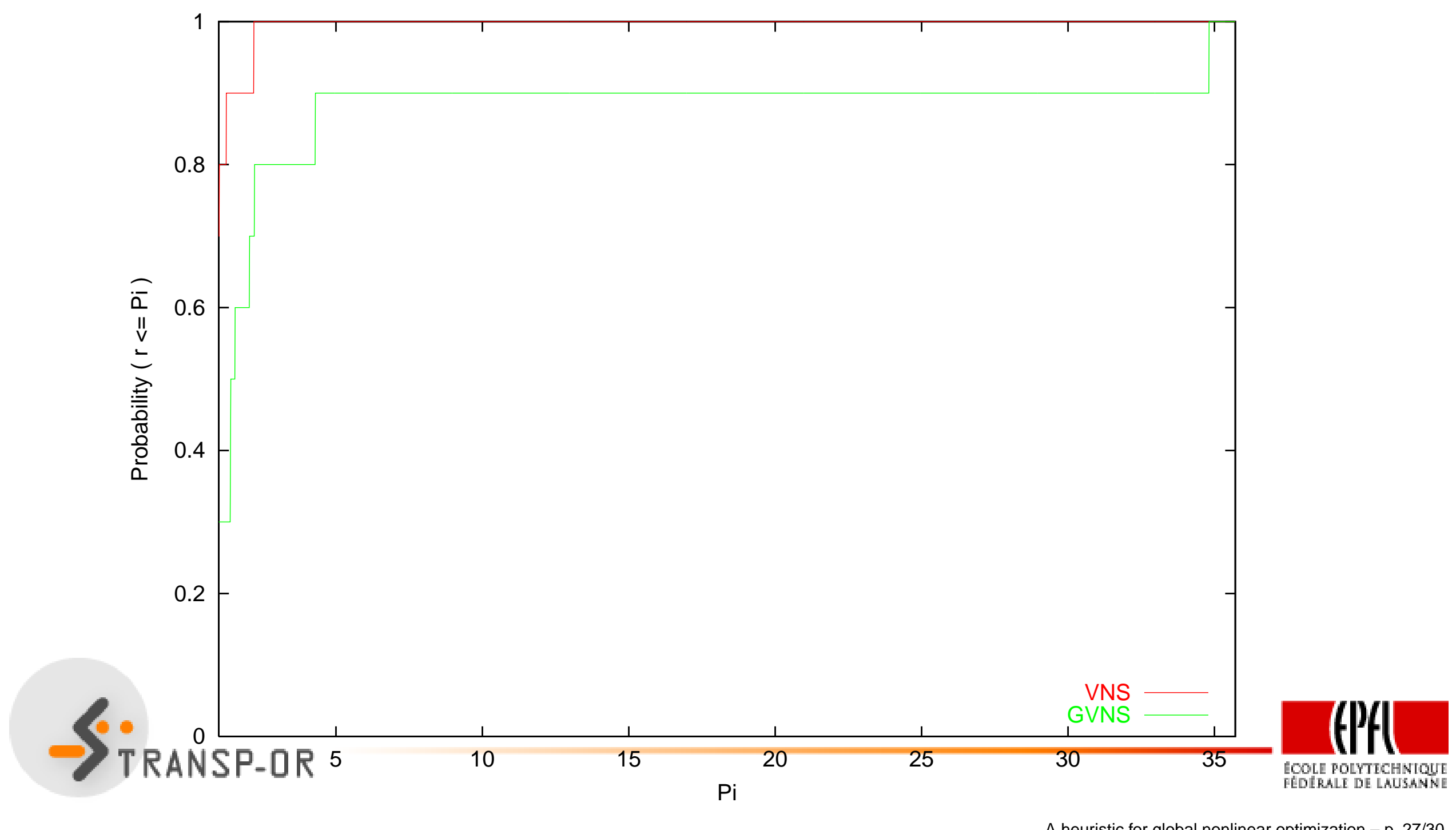




\section{Numerical results: efficiency}

Number of function evaluations (zoom)

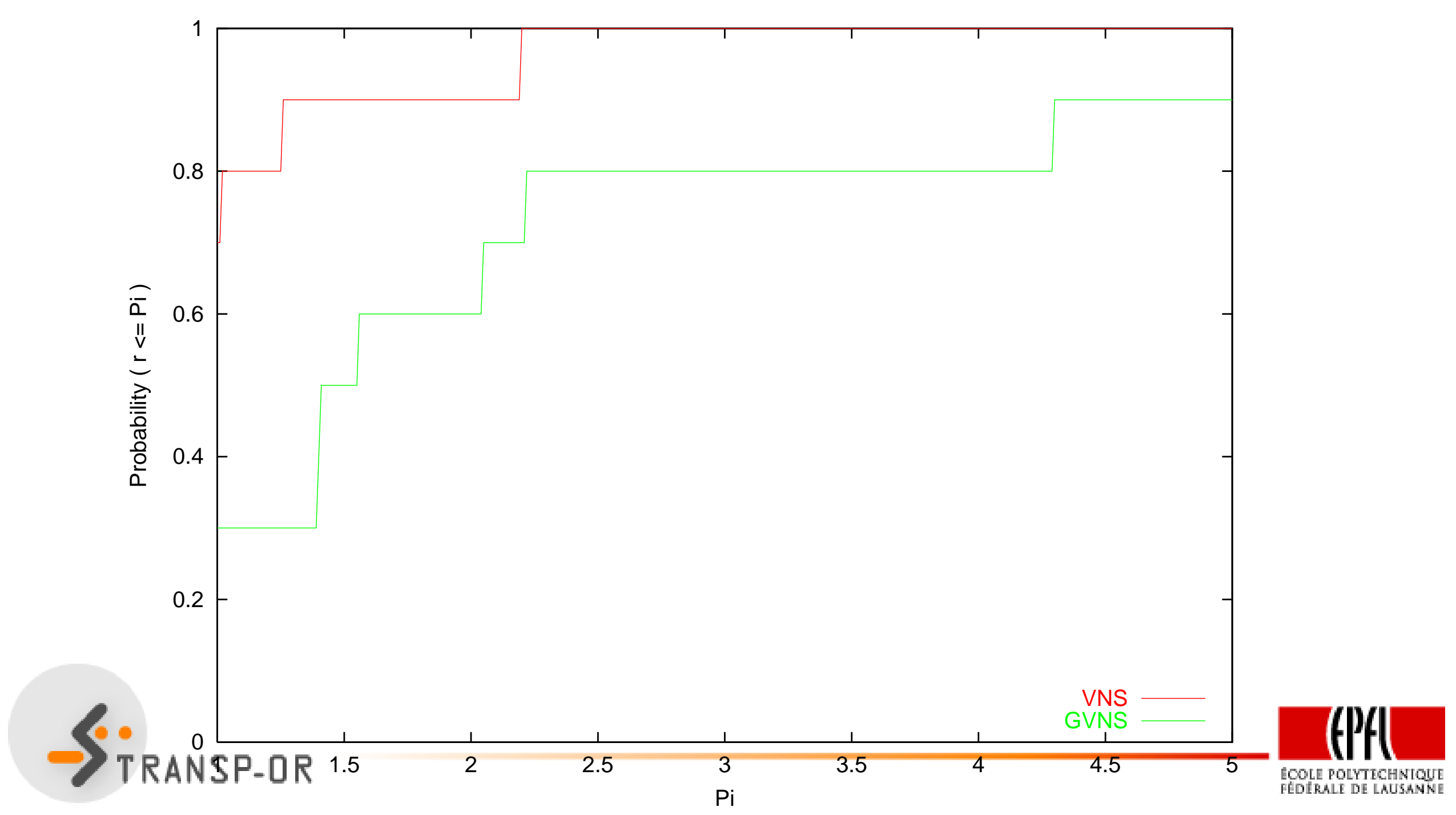




\section{Conclusions}

- Use of state of the art methods from

- nonlinear optimization: TR + Q-Newton

- discrete optimization: VNS

- Two new ingredients:

- Premature stop of LS to spare computational effort

- Exploits curvature for smart coverage

- Numerical results consistent with the algorithm design 


\section{Future work}

- Include preconditioning in the neighbor generation

- Exploit the structure of maximum likelihood estimation (Bastin et. al, 2006)

- Model of the convex hull of local optima 